\title{
RINRIA
}

\section{Aggregate and Fractal Tessellations}

\author{
Konstantin Tchoumatchenko*, Sergei Zuyev ${ }^{\dagger}$ \\ Thème 1 - Réseaux et systèmes \\ Projet Mistral \\ Rapport de recherche $\mathrm{n}^{\circ} 3699$ - Juin 1999 - 28 pages
}

\begin{abstract}
Consider a sequence of stationary tessellations $\left\{\Theta^{n}, n=0,1, \ldots\right\}$ of $R^{d}$ consisting of cells $\left\{C^{n}\left(x_{i}^{n}\right)\right\}$ with the nuclei $\left\{x_{i}^{n}\right\}$. An aggregate cell of level one, $C_{0}^{1}\left(x_{i}^{0}\right)$, is the result of merging the cells of $\Theta^{1}$ whose nuclei lie in $C^{0}\left(x_{i}^{0}\right)$. An aggregate tessellation $\Theta_{0}^{n}$ consists of the aggregate cells of level $n, C_{0}^{n}\left(x_{i}^{0}\right)$, defined recursively by merging those cells of $\Theta^{n}$ whose nuclei lie in $C_{0}^{n-1}\left(x_{i}^{0}\right)$.

We find an expression for the probability for a point to belong to a typical aggregate cell and obtain bounds for the probability of cell's expansion and extinction. We give necessary conditions for the limit tessellation to exist as $n \rightarrow \infty$ and provide upper bounds for the Hausdorff dimension of its fractal boundary and for the spherical contact distribution function in the case of Poisson-Voronoi tessellations $\left\{\Theta^{n}\right\}$.
\end{abstract}

Key-words: Aggregate tessellation - fractals - Voronoi tessellation - Poisson process Hausdorff measure - hierarchical models - telecommunications

\footnotetext{
* Ecole Normale Supérieure, DMI, 45 rue d'Ulm, 75230 Paris cedex 05, France. tchoumatchenko@ens.fr

$\dagger$ Statistics and Modelling Science dept., University of Strathclyde, 26 Richmond str., Glasgow, G1 1XH, UK. sergei@stams.strath.ac.uk
} 


\section{Tessellations Agrégées et Fractales}

Résumé : Soit $\left\{\Theta^{n}, n=0,1, \ldots\right\}$ une suite de tessellations stationnaires de $R^{d}$ constituées de cellules $\left\{C^{n}\left(x_{i}^{n}\right)\right\}$ avec des noyaux $\left\{x_{i}^{n}\right\}$. Une cellule agrégée du niveau un, $C_{0}^{1}\left(x_{i}^{0}\right)$, est le résultat de l'union des cellules de $\Theta^{1}$ dont les noyaux sont dans $C^{0}\left(x_{i}^{0}\right)$. Une tessellation agrégée $\Theta_{0}^{n}$ est composée des cellules agrégées du niveau $n, C_{0}^{n}\left(x_{i}^{0}\right)$, qui sont définies comme les unions des cellules de $\Theta^{n}$ dont les noyaux se trouvent dans $C_{0}^{n-1}\left(x_{i}^{0}\right)$, etc.

Nous obtenons une expression de la probabilité pour un point fixe d'être couvert par une cellule agrégée typique ainsi que les bornes sur les probabilités d'expansion et d'extinction d'une cellule. Nous donnons des conditions nécessaires de l'existence d'une tessellation limite quand $n \rightarrow \infty$. Dans le cas où $\left\{\Theta^{n}\right\}$ sont des tessellations de Poisson-Voronoï nous donnons également des bornes supérieures de la dimension de Hausdorff de sa frontière fractale et de la fonction de contacte sphérique.

Mots-clés : Tessellation agrégée - fractales - tessellation de Voronoï - processus de Poisson - mesure de Hausdorff - modèles hiérarchiques - télécommunications 


\section{Motivation}

A tessellation of $\mathbb{R}^{d}$ is a countable collection of closed Borel sets called cells such that

(a) union of all cells is the whole space;

(b) intersection of any two different cells has $d$-Lebesgue measure zero;

(c) each bounded set intersects a finite number of cells.

Tessellations are widely used to model different cellular systems: body's tissues in biology, granulated materials in material science, crystal arrangements in physics, etc (see, e. g., [10] and references therein).

Many examples of tessellations can be constructed by division of $\mathbb{R}^{d}$ between the elements of a countable nuclei set $\Pi=\left\{x_{i}\right\} \subset \mathbb{R}^{d}$ according to a certain rule. For example, the Voronoi tessellation has cells defined as

$$
C\left(x_{i}\right)=\left\{x \in \mathbb{R}^{d}:\left\|x-x_{i}\right\| \leq\left\|x-x_{j}\right\|, \forall j \neq i\right\},
$$

where $\|\cdot\|$ is the Euclidean norm. Thus, the cell with nucleus $x_{i}$ consists of the points that are closer to $x_{i}$ than to any other nucleus. Reciprocally, given a collection of cells, one can always assign a unique nucleus to each cell by a certain rule, for instance, its center of gravity. In this paper we deal with stationary tessellations with the cells being stationary random closed sets. We will always assume that the rule relating the cells with their nuclei set $\Pi$ satisfies an obvious compatibility condition:

$$
C\left(\theta\left(x_{i}\right), \theta(\Pi)\right)=\theta\left(C\left(x_{i}, \Pi\right)\right)
$$

for any shift transformation $\theta$ in $\mathbb{R}^{d}$ for which the point process $\Pi$ is itself stationary.

Recently, tessellations were used as models of service zones of telecommunication stations. Under the reasonable assumption that the network's subscribers are served by the nearest to them station and that the whole area is covered by the network's service, the zones form the Voronoi tessellation with the stations being the nuclei set. If the latter is modeled by a point process then the spatial variability of the zones and related performance characteristics of the network may be described in terms of functionals of the corresponding random sets and studied by means of stochastic analysis. The main advantage of these models is that they dramatically reduce the number of structuring parameters of the model to just a few parameters of the underlying stochastic process and often allow for an analytical treatment of complex networks characteristics (see [1], [2], [6]).

In many cases, however, models using Voronoi tessellation over-simplify the complex geometry of service zones. First of all, unlike Voronoi cells, real zones are not necessarily convex. The relative sizes of the zones are affected by capacities of different stations. In some cases, a station may not be contained in its proper zone. Last but not the least, the zones' boundaries may be very fuzzy as, for instance, in the case of wireless communications. In such systems, the base station that will handle a call from a mobile terminal is decided upon 
the signal's strength rather than Euclidean distance to the stations. Affected by the wave attenuation phenomena, the zones' boundaries have extremely irregular, distorted shapes.

This calls for development of more complex tessellation models that are still described in terms of a small number of parameters and simple enough to be analytically treatable.

For this we introduce an operation of aggregation on tessellations equipped with nuclei. Let $\Theta^{0}=\left\{C^{0}\left(x_{i}^{0}\right)\right\}$ and $\Theta^{1}=\left\{C^{1}\left(x_{i}^{1}\right)\right\}$ be two such tessellations. Define the cells of a new tessellation $\Theta_{0}^{1}=\Theta^{0} \circ \Theta^{1}$ as

$$
C_{0}^{1}\left(x_{i}^{0}\right)=\bigcup_{j: x_{j}^{1} \in C^{0}\left(x_{i}^{0}\right)} C^{1}\left(x_{j}^{1}\right) .
$$

In words, $C_{0}^{1}\left(x_{i}^{0}\right)$ is the union of all the cells of $\Theta^{1}$ whose nuclei lie in $C^{0}\left(x_{i}^{0}\right)$ (see Figure 1). The result of such operation is again a tessellation though some $C_{0}^{1}\left(x_{i}^{0}\right)$ may be empty. Due to the independence and stationarity assumptions, a.s. no $x_{j}^{1}$ lies on the boundary $\Gamma\left(\Theta^{1}\right)$, and hence each $\Theta^{1}$-cell belongs to a unique $\Theta_{0}^{1}$-cell. It is easy to verify that the operation of aggregation is associative

$$
\Theta^{0} \circ\left(\Theta^{1} \circ \Theta^{2}\right)=\left(\Theta^{0} \circ \Theta^{1}\right) \circ \Theta^{2}
$$

and that the aggregate tessellation is stationary provided the initial tessellations are stationary. Let $\left\{\Theta^{n}\right\}_{n \in \mathbb{N}}$ be a sequence of tessellations with the nuclei sets $\Pi_{n}=\left\{x_{i}^{n}\right\}, n \in \mathbb{N}$. Consequent aggregation of the first $n$ terms of the sequence yields the aggregate tessellation of order $n$

$$
\Theta_{0}^{n}=\Theta^{0} \circ \Theta^{1} \circ \ldots \circ \Theta^{n}
$$

with the nuclei set $\Pi_{0}=\left\{x_{i}^{0}\right\}$. The cells of this tessellation will be called aggregate $n$ cells and denoted by $C_{0}^{n}\left(x_{i}^{0}\right)$. Of course, aggregate cells constructed in this way depend on positions of the nuclei and are, in general, neither convex nor connected; nor do they need to contain the nucleus. Figure 2 shows several simulated aggregate cells with nucleus at the origin generated by independent Poisson-Voronoi plane tessellations with exponentially growing intensities.

It is clear that more the intensities of the consequent processes differ, less the boundary of cell $C_{0}^{n+1}\left(x_{i}^{0}\right)$ deviates from the boundary of $C_{0}^{n}\left(x_{i}^{0}\right)$. On the other hand, for close intensity values the boundary becomes very irregular, cells are more likely to split and quite often there is no points of $\Pi_{n+1}$ in $C_{0}^{n}\left(x_{i}^{0}\right)$, so that $C_{0}^{n+1}\left(x_{i}^{0}\right)$ is empty. Using an analogy with branching processes, we may think of the nuclei of the $\Theta^{n}$-cells that make up the aggregate cell $C_{0}^{n}\left(x_{i}^{0}\right)$, as of $n$-generation offspring of a 0-level parent nucleus $x_{i}^{0}$. If we connect by segments the nuclei $\Pi_{n}$ of each level $n$ with their offspring in the next level nuclei $\Pi_{n+1}$, we will obtain a family of spanning trees studied for Poisson-Voronoi case in [3]. In the present paper we address properties of the aggregate cells rather than those of the spanning trees.

New phenomena appear in the limit, when $n$ tends to infinity. As we have seen above, there exist models in which with positive probability some of the aggregate cells are empty, which means that the densities of 0 -level nuclei that have not died out till the $n$-th generation 

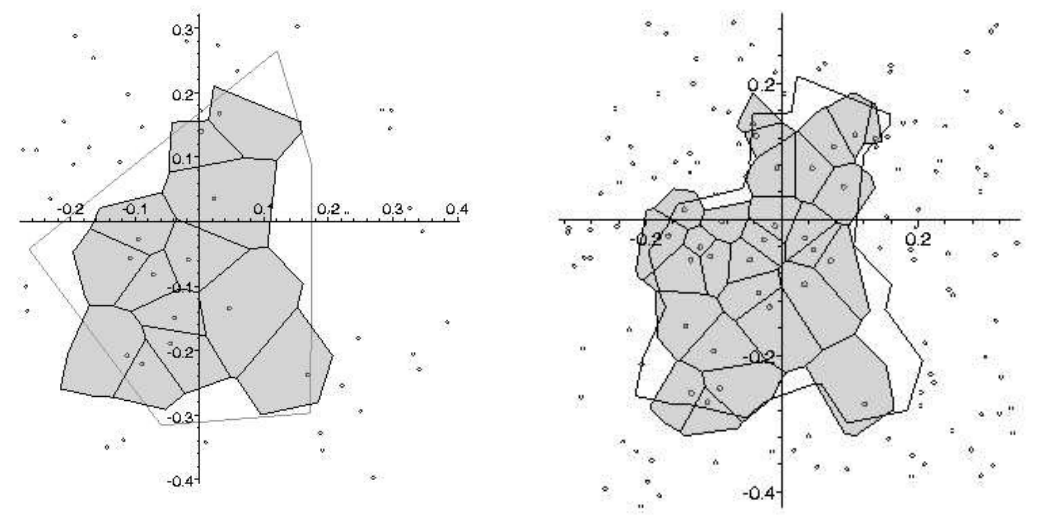

Figure 1: First and second iterations in the construction of aggregate cells. The boundary of the previous level cell is countered.
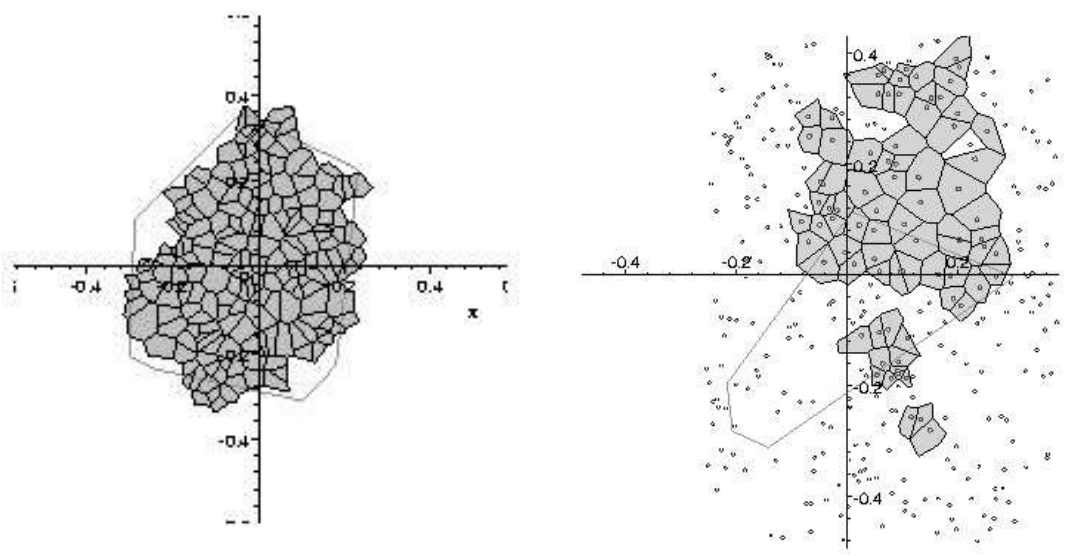

Figure 2: Aggregate cells $C_{0}^{n}(0)$ in PVAT model and the initial $C^{0}(0)$ Voronoi cell. Left image: $n=2, \lambda=10$. Right image: $n=20, \lambda=1.1$.

form a decreasing sequence. A priori it is not clear if the limit is positive, i.e., that we do not end up with all the 0 -nuclei $\Pi_{0}$ dying out with probability 1 . Next, even if the limit is positive, will $\Theta_{0}^{n}$ converge in some sense to a limit, say, $\Theta_{0}^{\infty}$ that is a tessellation? It is easy to imagine that $\Theta_{0}^{\infty}$ may have a fractal boundary (it also may not! - see Example 1 
in Section 2) and thus it is unclear if the boundary has $d$-Lebesgue measure 0 and whether only a finite number of the limit aggregate cells hits a bounded set. Finally, if the boundary does have a positive measure and thus the cells overlap, does a typical cell contain with a positive probability a "core" - an open set with all the points belonging only to this cell?

As the examples in the text readily show, different models may manifest very different behavior. We illustrate this on two basic models: cubic lattices and Poisson-Voronoi aggregate tessellations, quoted as PVAT in the sequel, for which the elements of the sequence $\left\{\Theta^{n}\right\}_{n \in \mathbb{N}}$ are all Voronoi tessellations generated, respectively, by mutually independent homogeneous Poisson point processes $\Pi_{n}, n \in \mathbb{N}$.

The structure of the paper is the following. In Section 2 we find an expression for the coverage probability function for a typical aggregate $n$-cell via the corresponding characteristics of $\Theta^{0}, \ldots, \Theta^{n}$. This result is valid for any independent sequence of stationary tessellations although a closed form expression can be obtained only in a few cases. In section 3 we find a uniform upper bound on the diameter of a typical cell in PVAT (a typical cell is the one with nucleus at the origin under the Palm distribution of the process $\Pi_{0}$ ). Here we also give estimates for the probability of cell's extinction and show that with positive probability there is a ball contained in all $n$-level aggregate cells $C_{0}^{n}(0)$. In the next Section we prove that this property is sufficient in general case for the limit cells, defined as the set lower limit of $\left\{C_{0}^{n}\left(x_{i}^{0}\right)\right\}_{n \in \mathbb{N}}$, to form a tessellation, so that their boundaries have almost surely $d$-Lebesgue measure 0. Defined by a simple recursive procedure, the boundary of the limit PVAT has an intricate auto-similar structure at any scale allowing us to call it fractal. To characterize its degree of irregularity, we provide in Section 6 an upper bound for its Hausdorff dimension. It is based on the analysis of the boundary's contact distribution function in the preceding Section 5. Note that the parts of the cell's fractal boundary are highly dependent making most of previously developed techniques for random fractals inapplicable in our case (a presentation of modern methods used in studying fractals can be found, e. g., in Kenneth Falconer's book [5]).

The following notation is used throughout the paper. By $\mathbf{P}$ we denote the distribution in a probability space carrying the sequence of independent stationary point processes $\Pi_{0}, \Pi_{1}, \ldots$, and by $\mathbf{P}_{n}^{0}$, the Palm distribution with respect to the process of nuclei $\Pi_{n}$ of level $n, n=0,1, \ldots$ Most frequently we consider the Palm distribution with respect to $\Pi_{0}$ for which we simply write $\mathbf{P}^{0}$ instead of $\mathbf{P}_{0}^{0}$. Similar notation is used for the corresponding expectations. $\Pi_{n}(B)$ stands for the number of points of $\Pi_{n}$ in a Borel set $B \subset \mathbb{R}^{d}$. The intensity of $\Pi_{n}$ is denoted by $\lambda_{n}$, so that $\mathbf{E}_{n} \Pi_{n}(B)=\lambda_{n}|B|$ and it is the only parameter characterizing a homogeneous Poisson process. We also assume that $\lambda_{0}=1$, which is just a matter of scale choice.

Finally,

$$
f * g(y)=\int_{\mathbb{R}^{d}} f(z) g(y-z) d z
$$


is the convolution of two functions, $b(x, r)$ is the closed ball centered in $x$ with radius $r$, and $b_{d}$ is the volume of a unit ball in $\mathbb{R}^{d}$ :

$$
b_{d}=\frac{\pi^{d / 2}}{\Gamma(d / 2+1)} .
$$

\section{Coverage probability}

Consider a tessellation $\Theta^{n}$ of fixed level $n$. Under Palm probability $\mathbf{P}_{n}^{0}$, there is almost surely a point of $\Pi_{n}$ at the origin 0 . Since the density of cells is $\lambda_{n}$, the volume of a typical cell is $\mathbf{E}_{n}^{0}\left|C^{n}(0)\right|=\lambda_{n}^{-1}$ (see, e. g., [9], Corollary 5.2, equation (5.6)). Therefore,

$$
\lambda_{n} \int \mathbf{P}_{n}^{0}\left\{y \in C^{n}(0)\right\} d y=\lambda_{n} \mathbf{E}_{n}^{0} \int \mathbb{I}\left(y \in C^{n}(0)\right) d y=\lambda_{n} \mathbf{E}_{n}^{0}\left|C^{n}(0)\right|=1,
$$

so that the function

$$
f_{n}(y)=\lambda_{n} \mathbf{P}_{n}^{0}\left\{y \in C^{n}(0)\right\}
$$

is a distribution density in $\mathbb{R}^{d}$. It has the following interpretation. Given a realization of $C^{n}(0)$, let $\xi_{n}$ be a r.v. uniformly distributed in the scaled set $\lambda_{n}^{1 / d} C^{n}(0)$. Then $f_{n}$ is the density of the the unconditional distribution of $\xi_{n}$ in $\mathbb{R}^{d}$.

The next statement provides a formula for calculating the probability that a point $y \in \mathbb{R}^{d}$ is covered by a typical aggregate cell of level $n$.

Theorem 1 Let $f_{0, n}(y)=\mathbf{P}^{0}\left\{y \in C_{0}^{n}(0)\right\}$. Then for each natural $n$,

$$
f_{0, n}(y)=f_{0} * f_{1} * \cdots * f_{n}(y) .
$$

Proof. By definition, for $n=0$, obviously,

$$
f_{0}(y)=\mathbf{P}^{0}\left\{y \in C^{0}(0)\right\} .
$$

Suppose, the statement of the Lemma holds for $n-1$. By the Campbell theorem (see, e. g., $[11$, p. 119]),

$$
\begin{aligned}
\mathbf{P}^{0}\left\{y \in C_{0}^{n}(0)\right\}=\mathbf{E}^{0} \sum_{x_{i}^{n} \in \Pi_{n}} \mathbb{I}\left(x_{i}^{n} \in\right. & \left.C_{0}^{n-1}(0)\right) \mathbb{I}\left(y \in C^{n}\left(x_{i}^{n}\right)\right) \\
& =\lambda_{n} \int_{\mathbb{R}^{d}} \mathbf{P}^{0}\left\{z \in C_{0}^{n-1}(0)\right\} \mathbf{P}_{n}^{0}\left\{y-z \in C^{n}(0)\right\} d z .
\end{aligned}
$$

It is easy to see that this expression is equivalent to

$$
f_{0, n}(y)=\int_{\mathbb{R}^{d}} f_{0, n-1}(z) f_{n}(y-z) d z .
$$


Example 1 Consider a stationary tessellation $\Theta^{n}$ of $\mathbb{R}^{d}$ obtained by shifting the regular mesh of $d$-hypercubes of side $\lambda_{n}^{-1}$ with a vertex at the origin by a random vector uniformly distributed in $\left[0, \lambda_{n}^{-1}\right]^{d}$. Note that the $d$-dimensional stationary mesh is simply a Cartesian product of $d$ one-dimensional independent components. Therefore, it is sufficient to study the case $d=1$ when $\Theta^{n}$ is a stationary sequence of the intervals of length $\lambda_{n}^{-1}$ on the line.

Assume that $\left\{\lambda_{n}\right\}$ is a non-increasing sequence and $\lambda_{0}=1$. It is easy to see by induction that the size of any $\Theta_{0}^{n}$-cell along each coordinate axis is at least $\lambda_{n}^{-1}$, so there is always at least one nucleus of $\Pi_{n+1}$ in each cell. As a result, all aggregate cells are rectangles and the cells never die. Moreover, for each $n$, the sizes of the aggregate cells along the line do not change if $\lambda_{n+1} / \lambda_{n}$ is a natural number and change periodically if $\lambda_{n+1} / \lambda_{n}$ is a rational one. By construction, the boundaries of the $\Theta^{n}$-cells have coordinates $\lambda_{n}^{-1}\left(k+u_{n}\right), k \in \mathbb{Z}$, where $u_{n}$ are independent uniformly distributed in $(0,1)$ random variables describing the shift. Define the nuclei set as

$$
\Pi_{n}=\left\{\lambda_{n}^{-1}\left(\alpha+k+u_{n}\right)\right\}, \quad k \in \mathbb{Z}, \alpha \in[0,1]
$$

so that the nucleus of each cell divides it in proportion $\alpha:(1-\alpha)$ from left to right. According to (1), the characteristic function of $f_{0}^{n}(y)$ is given by

$$
\chi_{0}^{n}(z)=\prod_{k=0}^{n} \frac{\lambda_{k}}{i z}\left(e^{i z \lambda_{k}^{-1}(1-\alpha)}-e^{-i z \lambda_{k}^{-1} \alpha}\right) .
$$

In fact, a complete analysis of this model is possible. Let $a_{k}^{n}$ be the right boundary point of the $n$-aggregate cell with the nucleus $\alpha+k+u_{0}, k \in \mathbb{Z}$. It is straightforward to verify that the evolution of the boundaries are given by the following recursion

$$
\begin{aligned}
& a_{k}^{n}=a_{k}^{n-1}+d_{k}^{n}, \quad \text { where } \\
& d_{k}^{n}=\lambda_{n}^{-1}\left(1-\alpha-\left\langle\lambda_{n} a_{k}^{n-1}-\alpha-u_{n}\right\rangle\right)
\end{aligned}
$$

with $\langle z\rangle=z-\max \{k \in \mathbb{Z}: k \leq z\}$ being the fractional part of a real number $z$. Note that for any $z$ and for any $u$ uniformly distributed in $(0,1)$, the r.v. $\langle z+u\rangle$ is again uniform in $(0,1)$. Therefore,

$$
\begin{aligned}
\mathbf{E} d_{k}^{n} & =\lambda_{n}^{-1}(1 / 2-\alpha) \\
\operatorname{var} d_{k}^{n} & =1 /\left(12 \lambda_{n}^{2}\right),
\end{aligned}
$$

so that there is a systematic drift to the right or to the left if $\alpha<1 / 2$ or $\alpha>1 / 2$, respectively. We have $\left|d_{k}^{n}\right| \leq 1$. By the well known theorem on random series convergence (see, e. g., [4, p. 239]), the boundaries of the aggregate cells almost surely stabilize as $n \rightarrow \infty$ if and only if both series $(1 / 2-\alpha) \sum_{n} \lambda_{n}^{-1}$ and $\sum_{n} \lambda_{n}^{-2}$ converge. We see a noticeable dependence on the nuclei choice when, say, $\lambda_{n}=n$. In this case the cells stabilize only if $\alpha=1 / 2$ and float to plus or minus infinity depending on whether $\alpha$ is smaller or greater than $1 / 2$. 
Let us take a closer look at the coverage probability for Poisson-Voronoi aggregate tessellations. For PVAT we have:

$$
f_{n}(y)=\lambda_{n} \exp \left\{-\lambda_{n} b_{d}\|y\|^{d}\right\} .
$$

The next two statements provide explicit formulas for the coverage probability in $\mathbb{R}^{1}$ and $\mathbb{R}^{2}$.

Example 2 Consider PVAT in $\mathbb{R}^{1}$ and assume that all $\lambda_{i}$ are pairwise different. Then

$$
f_{0, n}(y)=\sum_{i=0}^{n} c_{i} \lambda_{i} \exp \left\{-2 \lambda_{i}|y|\right\}
$$

where

$$
c_{i}=2 \prod_{\substack{l=0 \\ l \neq i}}^{n}\left(1-\frac{\lambda_{i}}{\lambda_{l}}\right)^{-1} \sum_{j=0}^{n}\left(1+\frac{\lambda_{i}}{\lambda_{j}}\right)^{-1} \prod_{\substack{m=0 \\ m \neq j}}^{n}\left(1-\frac{\lambda_{j}}{\lambda_{m}}\right)^{-1}
$$

Indeed, from Theorem 1 it follows that $f_{0, n}(y)$ is the density of the sum of independent r.v.'s $\sum_{i=0}^{n} \xi_{i}$ whose densities $f_{i}(y)$ are given by (2) with $d=1$. Note that $\xi_{i}=\nu_{i}-\nu_{i}^{\prime}$, where $\nu_{i}, \nu_{i}^{\prime}$ are independent exponentially distributed r.v.'s with parameter $2 \lambda_{i}$. The density of $\sum_{i=0}^{n} \nu_{i}$ equals (see, e. g., [4, p. 170])

$$
\sum_{i=0}^{n} 2 \lambda_{i} \exp \left\{-2 \lambda_{i} y\right\} \prod_{\substack{l=0 \\ l \neq i}}^{n}\left(1-\frac{\lambda_{i}}{\lambda_{l}}\right)^{-1} \mathbb{I}(y \geq 0) .
$$

Finding its symmetrization, we obtain (3).

Example 3 Consider PVAT in $\mathbb{R}^{2}$. According to (2), in $\mathbb{R}^{2}$ the r.v. $\xi_{i}$ has normal distribution with zero mean and the covariance matrix

$$
\left(\begin{array}{cc}
\left(2 \pi \lambda_{i}\right)^{-1} & 0 \\
0 & \left(2 \pi \lambda_{i}\right)^{-1}
\end{array}\right)
$$

Thus $\sum_{i=0}^{n} \xi_{i}$ is also normal with zero mean and the covariance matrix being the sum of (4):

$$
\left(\begin{array}{cc}
\left(2 \pi L_{n}\right)^{-1} & 0 \\
0 & \left(2 \pi L_{n}\right)^{-1}
\end{array}\right)
$$

where

$$
L_{n}=\left(\sum_{i=0}^{n} \frac{1}{\lambda_{i}}\right)^{-1}
$$

$\mathrm{RR} \mathrm{n}^{\circ} 3699$ 
The corresponding density is therefore

$$
f_{0, n}(y)=L_{n} \exp \left\{-L_{n} \pi\|y\|^{2}\right\}
$$

that is the same as for a typical cell in the ordinary Voronoi tessellation with the nuclei intensity $L_{n}$. Such "mean field" approximation is valid only in this planar case, the reason being the stability of the distributions of $\xi_{i}$ 's in $d=2$.

Example 4 For PVAT in $\mathbb{R}^{d}$, as follows from (1), the characteristic function of $f_{0, n}(y)$ is given by

$$
\prod_{n} \chi\left(t / \lambda_{n}^{-1 / d}\right)
$$

where $\chi(t)$ is the characteristic function of the density $f_{0}(y)$ in (2). It can be shown that

$$
\chi(t)=\frac{\Gamma\left(\frac{d}{2}\right)}{\pi^{1 / 2}} \sum_{m=0}^{\infty} \frac{(-1)^{m}}{(2 m) !} \frac{\|t\|^{2 m}}{\pi^{m}}\left[\Gamma\left(1+\frac{d}{2}\right)\right]^{2 m / d} \frac{\Gamma\left(m+\frac{1}{2}\right) \Gamma\left(1+\frac{2 m}{d}\right)}{\Gamma\left(m+\frac{d}{2}\right)} .
$$

An alternative representation uses Bessel functions of the first kind (see, e. g., [7]):

$$
\chi(t)=\frac{(2 \pi)^{d / 2}}{\|t\|^{d / 2-1}} \int_{0}^{\infty} \rho^{d / 2} e^{-b_{d} \rho^{d}} J_{d / 2-1}(\rho\|t\|) d \rho .
$$

\section{Evolution of aggregate cells}

In this section we will investigate the behavior of the typical aggregate cell $C_{0}^{n}(0)$ as $n$ tends to infinity on the Palm space of the process $\Pi_{0}$. The maximal and the minimal distance from a point $z$ to the cell's boundary can be defined, respectively, as

$$
\begin{aligned}
& R_{n}(z)= \begin{cases}\min \left\{r: b(z, r) \supset C_{0}^{n}(0)\right\} & \text { if } C_{0}^{n}(0) \neq \emptyset, \\
0 & \text { otherwise }\end{cases} \\
& r_{n}(z)= \begin{cases}\max \left\{r: b(z, r) \subset C_{0}^{n}(0)\right\} & \text { if } z \in C_{0}^{n}(0), \\
0 & \text { otherwise }\end{cases}
\end{aligned}
$$

The definition takes into account the fact that the aggregate cell of order $n \geq 1$ might not contain $z$ or might be empty. Our aim is to characterize the distribution of

$$
\begin{gathered}
R_{\infty}(z)=\sup _{n} R_{n}(z), \\
r_{\infty}(z)=\inf _{n} r_{n}(z) .
\end{gathered}
$$


Theorem 2 Let $\phi(y)$ be the inverse of the function $y(x)=x e^{x}$. Assume that

$$
c=\sum_{n=1}^{\infty}\left(\frac{\phi\left(\lambda_{n}\right)}{\lambda_{n}}\right)^{1 / d}=\sum_{n=1}^{\infty} e^{-\phi\left(\lambda_{n}\right) / d}<\infty
$$

Then for PVAT the following inequalities hold for all $\rho>c \sqrt{d}$ and any $z \in \mathbb{R}^{d}$ :

$$
\begin{aligned}
& \mathbf{P}^{0}\left\{R_{\infty}(z)>\rho+\|z\|\right\} \leq a_{1} \sum_{n=1}^{\infty} e^{-\phi\left(\lambda_{n}\right) A(\rho)}, \\
& \mathbf{P}^{0}\left\{r_{\infty}(z)=0 \mid r_{0}(z)>\rho\right\} \leq a_{1} \sum_{n=1}^{\infty} e^{-\phi\left(\lambda_{n}\right) A(\rho)},
\end{aligned}
$$

where

$$
\begin{aligned}
& a_{1}=2 b_{d} d^{d / 2+1} c^{d-1}, \\
& A(\rho)=\left(\frac{\rho}{c \sqrt{d}}\right)^{d}+1 / d-1 .
\end{aligned}
$$

Proof. We begin from inequality (6). Since

$$
\mathbf{P}^{0}\left\{R_{\infty}(z)>\rho+\|z\|\right\} \leq \mathbf{P}^{0}\left\{R_{\infty}(0)>\rho\right\}
$$

it is sufficient to prove (6) for $z=0$. Let $\left\{\rho_{n}\right\}$ be a monotonously increasing sequence of positive numbers converging to $\rho$, then

$$
\left\{R_{\infty}(0)>\rho\right\} \subset \cup_{n=0}^{\infty}\left\{R_{n}(0)>\rho_{n}\right\} .
$$

Next, we use the following inequality: if $B \subset \cup_{n=0}^{\infty} B_{n}$, then

$$
\mathbf{P}(B) \leq \mathbf{P}\left(B_{0}\right)+\sum_{n=0}^{\infty} \mathbf{P}\left(B_{n} \cap \bar{B}_{n-1}\right) .
$$

Hence,

$$
\mathbf{P}^{0}\left\{R_{\infty}(0)>\rho\right\} \leq \mathbf{P}^{0}\left\{R_{0}>\rho_{0}\right\}+\sum_{n=0}^{\infty} \mathbf{P}^{0}\left\{R_{n}(0)>\rho_{n}, R_{n-1}(0) \leq \rho_{n-1}\right\} .
$$

The event

$$
\left\{R_{n}(0)>\rho_{n}, R_{n-1}(0) \leq \rho_{n-1}\right\}
$$

implies the existence of a Voronoi cell $C^{n}\left(x_{i}^{n}\right)$ with the nucleus inside of the ball $b\left(0, \rho_{n-1}\right)$ containing some point $y$ on the sphere $\partial b\left(0, \rho_{n}\right)$. Therefore, the interior of the ball $b(y, \| y-$ $\left.x_{i}^{n} \|\right)$ contains no points of $\Pi_{n}$ (see Figure 3 ). 


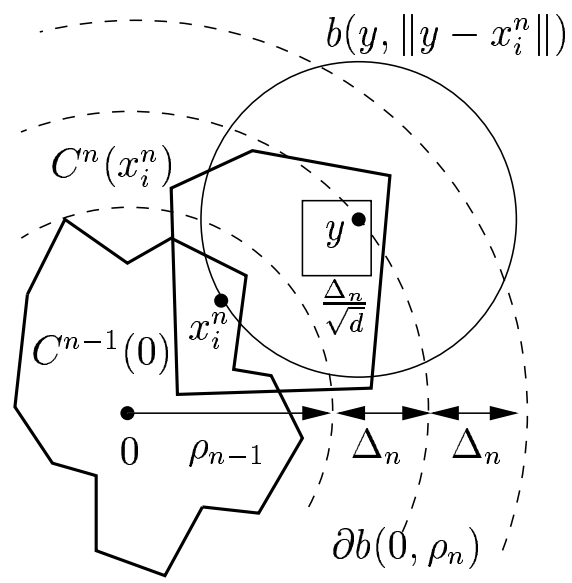

Figure 3: Large increase in $R_{n}(0)$ implies existence of a large empty ball.

Denote $\Delta_{n}=\rho_{n}-\rho_{n-1}$ and consider the collection of mesh cubes of side $\Delta_{n} / \sqrt{d}$ that lie entirely in the annulus $b\left(0, \rho_{n}+\Delta_{n}\right) \backslash b\left(0, \rho_{n-1}\right)$. Denote by $N$ their number. At least one of the mesh cubes lies entirely in the ball $b\left(y,\left\|y-x_{i}^{n}\right\|\right)$ (e.g., the cube containing $y$ ). Thus for the summands in (8) we obtain a bound, which is the probability that at least one of the mesh cubes contains no points of $\Pi_{n}$

$$
1-\left(1-\exp \left\{-\lambda_{n}\left(\Delta_{n} / \sqrt{d}\right)^{d}\right\}\right)^{N} .
$$

Write the corresponding inequality for the volumes of the union of the cubes and the volume of the annulus:

$$
\begin{aligned}
N\left(\frac{\Delta_{n}}{\sqrt{d}}\right)^{d} & <b_{d}\left[\left(\rho_{n}+\Delta_{n}\right)^{d}-\left(\rho_{n}-\Delta_{n}\right)^{d}\right] \\
& =2 b_{d} \Delta_{n} \sum_{k=0}^{d-1}\left(\rho_{n}+\Delta_{n}\right)^{k}\left(\rho_{n}-\Delta_{n}\right)^{d-k-1} \\
& <2 d b_{d} \Delta_{n} \sum_{k=0}^{d-1} \rho^{k} \rho^{d-k-1}<2 d b_{d} \Delta_{n} \rho^{d-1}
\end{aligned}
$$

This yields

$$
N<2 b_{d} d^{d / 2+1}\left(\frac{\rho}{\Delta_{n}}\right)^{d-1} .
$$

Since for any $0<a<1$ and $N \geq 1$,

$$
1-(1-a)^{N}<a N
$$


the value of (9) does not exceed

$$
2 b_{d} d^{d / 2+1}\left(\frac{\rho}{\Delta_{n}}\right)^{d-1} \exp \left\{-\lambda_{n}\left(\Delta_{n} / \sqrt{d}\right)^{d}\right\} .
$$

Next, choose a special sequence $\left\{\rho_{n}\right\}$ with the increments $\Delta_{n}$ given by

$$
\Delta_{n}=\frac{\rho}{c} e^{-\phi\left(\lambda_{n}\right) / d}
$$

with $c$ defined in (5). It is easy to see that $\rho_{n}$ monotonously converges to $\rho$. For such choice of $\left\{\rho_{n}\right\}$, from (10) it follows that the right-hand side of (8) is bounded by

$$
2 b_{d} d^{d / 2+1} \sum_{n=0}^{\infty} \exp \left\{-\left(\frac{\rho}{c \sqrt{d}}\right)^{d} \phi\left(\lambda_{n}\right)+(d-1)\left(\log c+\frac{\phi\left(\lambda_{n}\right)}{d}\right)\right\},
$$

which is equivalent to (6). We have used here the definition of $\phi$, due to which $e^{-\phi\left(\lambda_{n}\right)}=$ $\phi\left(\lambda_{n}\right) / \lambda_{n}$. The function $A(\rho)=O\left(\rho^{d}\right)$ increases to infinity and is greater than $1 / d$ for all $\rho>c \sqrt{d}$. Therefore, for such $\rho$, the series in (6) converges and the whole bound tends to 0 as $\rho \rightarrow \infty$ providing the almost sure finiteness of $R_{\infty}(z)$.

Inequality (7) is proved much in the same manner. Fix a small $0<\varepsilon<1$ and consider a sequence $\left\{\rho_{n}^{\prime}\right\}$ with $\rho_{0}^{\prime}=\rho$ that monotonously decreases to $\varepsilon \rho$. First, from

$$
\left\{r_{\infty}(z)=0, r_{0}(z)>\rho\right\} \subset \cup_{n=1}^{\infty}\left\{r_{n}(z)<\rho_{n}^{\prime}, r_{0}(z)>\rho\right\}
$$

it follows that

$$
\mathbf{P}^{0}\left\{r_{\infty}(z)=0 \mid r_{0}(z)>\rho\right\} \leq \sum_{n=1}^{\infty} \mathbf{P}^{0}\left\{r_{n}(z)<\rho_{n}^{\prime}, r_{n-1}(z) \geq \rho_{n-1}^{\prime} \mid r_{0}(z)>\rho\right\} .
$$

The event

$$
\left\{r_{n}(z)<\rho_{n}^{\prime}, r_{n-1}(z) \geq \rho_{n-1}^{\prime}\right\}
$$

implies the existence of a Voronoi cell $C^{n}\left(x_{i}^{n}\right)$ with the nucleus outside of the ball $b\left(z, \rho_{n-1}^{\prime}\right)$ having some point $y \in C^{n}\left(x_{i}^{n}\right)$ inside $b\left(z, \rho_{n}^{\prime}\right)$. Hence, there exists a ball of radius at least $\Delta_{n}^{\prime}=\rho_{n-1}^{\prime}-\rho_{n}^{\prime}$ centered on the sphere $\partial b\left(z, \rho_{n-1}^{\prime}\right)$. Note that this event is independent of the event $\left\{r_{0}(z)>\rho\right\}$. Thus the summands in (12) can be bounded as in (9) with $\Delta_{n}^{\prime}=(1-\varepsilon) \Delta_{n}$. With that choice of $\Delta_{n}^{\prime}$ the right hand side of (12) is bounded by an expression similar to $(11)$ with $c$ replaced by $(1-\varepsilon) c$. Due to the arbitrariness of $\varepsilon$, expression (11) also provides an upper bound. The rest of the proof remains unchanged.

Remark 1 By definition, $\log \phi(x)+\phi(x)=\log x$. Therefore, for $x \geq 1$ one has $\phi(x) \leq \log x$. Since $e^{-\phi\left(\lambda_{n}\right)}=\phi\left(\lambda_{n}\right) / \lambda_{n}$ and $\lambda_{n}>1$ for all sufficiently large $n$, the condition

$$
\sum_{n=1}^{\infty}\left(\frac{\log \lambda_{n}}{\lambda_{n}}\right)^{1 / d}<\infty \quad \text { or } \quad \sum_{n=1}^{\infty} \lambda_{n}^{1 / d-\varepsilon}<\infty
$$


for some $0<\varepsilon<1 / d$ is sufficient for (5) to hold. Moreover, $\phi(x) \geq(1+\log x) / 2$ for all $x$. Thus

$$
a_{1} \sum_{n=1}^{\infty} e^{-\phi\left(\lambda_{n}\right) A(\rho)} \leq a_{1} e^{-A(\rho) / 2} \sum_{n=1}^{\infty} \lambda_{n}^{-A(\rho) / 2}
$$

and the last function can also be used in (6-7).

Corollary 1 For PVAT with exponentially growing intensities $\lambda_{n}=\lambda^{n}$ for some $\lambda>1$, one has for all $\rho>c_{*}$ and any $z \in \mathbb{R}^{d}$,

$$
\begin{aligned}
& \mathbf{P}^{0}\left\{R_{\infty}(z)>\rho+\|z\|\right\} \leq c_{1} \exp \left\{-c_{2} \rho^{d}\right\}, \\
& \mathbf{P}^{0}\left\{r_{\infty}(z)=0 \mid r_{0}(z)>\rho\right\} \leq c_{1} \exp \left\{-c_{2} \rho^{d}\right\} .
\end{aligned}
$$

One may take

$$
\begin{aligned}
& c_{1}=a_{1}\left(1+d \frac{1+\phi(\lambda)}{\phi(\lambda) \log \lambda}\right) e^{\phi(\lambda)} \\
& c_{2}=\phi(\lambda)(c \sqrt{d})^{-d} \\
& c_{*}=\left(\left(\log c_{1}\right) / c_{2}\right)^{1 / d}
\end{aligned}
$$

Proof. Recall the following integral estimate:

$$
\sum_{n=1}^{\infty} h(n) \leq h(1)+\int_{1}^{\infty} h(x) d x
$$

for any non-increasing positive function $h(x)$. We have

$$
\begin{aligned}
\sum_{n=1}^{\infty} e^{-\phi\left(\lambda^{n}\right) A(\rho)} \leq e^{-\phi(\lambda) A(\rho)}+\int_{1}^{\infty} e^{-\phi\left(\lambda^{x}\right) A(\rho)} d x & \\
& =e^{-\phi(\lambda) A(\rho)}+\frac{1}{\log \lambda} \int_{\phi(\lambda)}^{\infty}\left(1+y^{-1}\right) e^{-y A(\rho)} d y
\end{aligned}
$$

after the variable change $y=\phi\left(\lambda^{x}\right)$. Next, since $1+y^{-1} \leq 1+\phi(\lambda)^{-1}$ on the integration domain, the whole expression can be bounded by

$$
e^{-\phi(\lambda) A(\rho)}+\frac{1+\phi(\lambda)^{-1}}{A(\rho) \log \lambda} e^{-\phi(\lambda) A(\rho)}<\left(1+\frac{1+\phi(\lambda)^{-1}}{d^{-1} \log \lambda}\right) e^{-\phi(\lambda) A(\rho)},
$$

so that (6-7) become (13-14), respectively. It can be immediately verified that these estimates become nontrivial if $\rho>c_{*}$ and that $c_{*}>c \sqrt{d}$. 
Corollary 2 For PVAT with polynomially growing intensities $\lambda_{n}=(1+\alpha n)^{\beta}$ for some $\alpha>0, \beta>d$, one has

$$
\sum_{n=1}^{\infty} e^{-\phi\left(\lambda_{n}\right) A(\rho)} \leq \frac{A(\rho)}{A(\rho)-\beta^{\prime}}\left(e^{-\phi\left(\lambda_{1}\right) A(\rho)}+\frac{\Gamma\left(\beta^{\prime}, \phi\left(\lambda_{1}\right)\left(A(\rho)-\beta^{\prime}\right)\right)}{\alpha \beta\left(A(\rho)-\beta^{\prime}\right)^{\beta^{\prime}}}\right),
$$

where $\beta^{\prime}=\beta^{-1}$ and $\Gamma(n, x)=\int_{x}^{\infty} z^{n-1} e^{-z} d z$ is the incomplete Gamma-function.

Proof. is the same as in Corollary 1 with the variable change $z=\left(A(\rho)-\beta^{-1}\right) \phi\left((1+\alpha x)^{\beta}\right)$ in the corresponding integral.

Corollary 3 Under conditions of Theorem 2, with probability one, each family of cells $\left\{C_{0}^{n}\left(x_{i}^{0}\right)\right\}_{n \in \mathbb{N}}$ is uniformly bounded in $\mathbb{R}^{d}$.

Proof. Let $\tau_{x}$ be the stationary shift defined on the probability space $\Omega$ such that

$$
\Pi_{n}\left(\tau_{x} \omega\right)(B)=\Pi_{n}(\omega)(B-x)
$$

for any Borel set $B \subset \mathbb{R}^{d}$ and any $n$. In this notation, $\tau_{x_{i}^{0}} R_{n}(0)$ for $x_{i}^{0} \in \Pi_{0}$ is the maximal distance from $x_{i}^{0}$ to the boundary of $C_{0}^{n}\left(x_{i}^{0}\right)$ that corresponds to the above definition of $R_{n}(0)$ with $C_{0}^{n}(0)$ replaced with $C_{0}^{n}\left(x_{i}^{0}\right)=\tau_{x_{i}^{0}} C_{0}^{n}(0)$. The probability that there exists an unbounded family of cells with the nucleus in a ball $b(0, N)$ equals

$$
\begin{aligned}
\mathbf{P} \bigcup_{x_{i} \in \Pi_{0} \cap b(0, N)}\left\{\tau_{x_{i}^{0}} R_{\infty}(0)\right. & =\infty\} \\
\leq \mathbf{E} \sum_{x_{i} \in \Pi_{0} \cap b(0, N)} \mathbb{I}\left\{\tau_{x_{i}^{0}} R_{\infty}(0)=\infty\right\} & =b_{d} N^{d} \mathbf{E}^{0} \mathbb{I}\left\{R_{\infty}(0)=\infty\right\}=0,
\end{aligned}
$$

where we have used the Campbell theorem and (6). Letting $N$ grow to infinity proves the assertion.

The following fact will be used later to show that the boundaries of the limit tessellation have zero Lebesgue measure.

Corollary 4 Under conditions of Theorem 2 , for any $y \in \mathbb{R}^{d}$ with positive probability, there exists $x_{i}^{0} \in \Pi_{0}$ such that

$$
y \in \operatorname{int}\left(\cap_{n} C_{0}^{n}\left(x_{i}^{0}\right)\right) .
$$

The lower bound for such probability is given in (17).

$\mathrm{RR} \mathrm{n}^{\circ} 3699$ 
Proof. Indeed, it is sufficient that the distance from $y$ to the boundary of the $\Pi_{0}$-cell containing $y$ is sufficiently large so that the boundaries of the progressing $n$-cells never reach $y$. The probability of the latter event is positive by (7).

Due to stationarity, we may put $y=0$. Consider the following representation:

$$
\mathbf{P}\left\{\exists x_{i}^{0}: 0 \in \operatorname{int}\left(\cap_{n} C_{0}^{n}\left(x_{i}^{0}\right)\right)\right\}=\mathbf{E} \sum_{x_{i}^{0} \in \Pi_{0}} \mathbb{I}\left(0 \in \operatorname{int}\left(\cap_{n} C_{0}^{n}\left(x_{i}^{0}\right)\right)\right) .
$$

By the Campbell theorem and by the isotropy, the right-hand side equals

$$
\begin{aligned}
& \int_{\mathbb{R}^{d}} \mathbf{P}^{0}\left\{-z \in \operatorname{int}\left(\cap_{n} C_{0}^{n}(0)\right)\right\} d z= \\
& \qquad \int_{\mathbb{R}^{d}} \mathbf{P}^{0}\left\{z \in \operatorname{int}\left(\cap_{n} C_{0}^{n}(0)\right) \mid b(z, \rho) \subset C^{0}(0)\right\} \\
& \quad \times \mathbf{P}^{0}\left\{b(z, \rho) \subset C^{0}(0)\right\} d z
\end{aligned}
$$

for arbitrary $\rho>0$. By Theorem 2, the first factor under the integral in (15) is greater than $1-a_{1} \sum_{n=1}^{\infty}\left(\phi\left(\lambda_{n}\right) / \lambda_{n}\right)^{A(\rho)}$ provided that $\rho \geq c \sqrt{d}$. The second factor equals the probability that no points of $\Pi_{0}$ lie in the figure $\cup_{\left\|z-z^{\prime}\right\|=\rho} b\left(z^{\prime},\left\|z^{\prime}\right\|\right)$. This figure is obtained by rotation of a cardioid around its symmetry axis; by construction, it is contained in the ball $b(z,\|z\|+2 \rho)$. Therefore,

$$
\mathbf{P}^{0}\left\{b(z, \rho) \subset C^{0}(0)\right\}>\exp \left\{-b_{d}(\|z\|+2 \rho)^{d}\right\} .
$$

Using this estimate we get

$$
\begin{aligned}
\int_{\mathbb{R}^{d}} \mathbf{P}^{0}\left\{b(z, \rho) \subset C^{0}(0)\right\} d z & >\int_{\mathbb{R}^{d}} \exp \left\{-b_{d}(\|z\|+2 \rho)^{d}\right\} d z \\
& >\int_{0}^{\rho} r^{d-1} d b_{d} \exp \left\{-b_{d}(3 \rho)^{d}\right\} d r=b_{d} \rho^{d} \exp \left\{-b_{d}(3 \rho)^{d}\right\} .
\end{aligned}
$$

Hence

$$
\begin{aligned}
\mathbf{P}\left\{\exists x_{i}^{0}: y \in \operatorname{int}(\right. & \left.\left.\cap_{n} C_{0}^{n}\left(x_{i}^{0}\right)\right)\right\} \\
& >\sup _{\rho \geq c \sqrt{d}}\left[b_{d} \rho^{d} \exp \left\{-b_{d}(3 \rho)^{d}\right\}\left(1-a_{1} \sum_{n=1}^{\infty}\left(\frac{\phi\left(\lambda_{n}\right)}{\lambda_{n}}\right)^{A(\rho)}\right)\right]>0 .
\end{aligned}
$$

The following corollary gives bounds for the probability of a cell's extinction.

Corollary 5 Under conditions of Theorem 2 , for any $1 \leq N \leq \infty$ one has $0<\mathbf{P}^{0}\left\{C_{0}^{N}(0)=\right.$ $\emptyset\}<1$. The corresponding bounds for the exponential case $\lambda_{n}=\lambda^{n}$ are given by (19) and (20) below. 
Proof. Obviously the cell centered in the origin dies on level $n$ if no points of $\Pi_{n}$ fall into $b\left(0, R_{n-1}(0)\right)$. Therefore,

$$
\begin{aligned}
\mathbf{P}^{0}\left\{C_{0}^{N}(0)=\emptyset\right\} \geq \sup _{1 \leq n \leq N} & \mathbf{P}^{0}\left\{C_{0}^{n}(0)=\emptyset\right\} \\
\geq & \sup _{1 \leq n \leq N} \mathbf{P}^{0}\left\{\Pi_{n}\left(b\left(0, R_{n-1}(0)\right)\right)=0\right\} \\
& =\sup _{1 \leq n \leq N} \mathbf{E}^{0} \exp \left\{-b_{d} \lambda_{n} R_{n-1}^{d}(0)\right\} \\
& =\sup _{1 \leq n \leq N}\left[1-\int_{0}^{\infty} d b_{d} \lambda_{n} r^{d-1} \exp \left\{-b_{d} \lambda_{n} r^{d}\right\} \mathbf{P}^{0}\left\{R_{n-1}(0)>r\right\} d r\right] .
\end{aligned}
$$

By Theorem 2, for $r \geq c \sqrt{d}$,

$$
\mathbf{P}^{0}\left\{R_{n-1}(0)>r\right\} \leq \mathbf{P}^{0}\left\{R_{\infty}(0)>r\right\} \leq a_{1} \sum_{n=1}^{\infty}\left(\frac{\phi\left(\lambda_{n}\right)}{\lambda_{n}}\right)^{A(r)} .
$$

Choose $r_{*}>c \sqrt{d}$ such that the last expression is less than 1 for all $r>r_{*}$. Then we obtain the following estimate

$$
\begin{aligned}
\mathbf{P}^{0}\left\{C_{0}^{n}(0)=\emptyset\right\} \geq 1-\int_{0}^{r_{*}} d b_{d} r^{d-1} \lambda^{n} \exp \left\{-b_{d} \lambda^{n} r^{d}\right\} d r & \\
& -\int_{r_{*}}^{\infty} d b_{d} r^{d-1} \lambda^{n} a_{1} \sum_{n=1}^{\infty}\left(\frac{\phi\left(\lambda_{n}\right)}{\lambda_{n}}\right)^{A(r)} d r
\end{aligned}
$$

which can be worked out in each particular case. For instance, in the exponential case considered in Corollary 1, with $r_{*}=c_{*}$ and (13) we have

$$
\begin{aligned}
\mathbf{P}^{0}\left\{C_{0}^{n}(0)=\emptyset\right\} \geq 1-\int_{0}^{c_{*}} d b_{d} r^{d-1} \lambda^{n} & \exp \left\{-b_{d} \lambda^{n} r^{d}\right\} d r \\
& -\int_{c_{*}}^{\infty} d b_{d} r^{d-1} \lambda^{n} c_{1} \exp \left\{-\left(b_{d} \lambda^{n}+c_{2}\right) r^{d}\right\} d r .
\end{aligned}
$$

Calculating the integrals in (18) and summing up the results, we obtain the estimate

$$
\mathbf{P}^{0}\left\{C_{0}^{N}(0)=\emptyset\right\} \leq \sup _{1 \leq n \leq N}(2 \lambda)^{-b_{d} \lambda^{n} / c_{2}}\left[1-\frac{2 d b_{d}^{2} \lambda^{n-1}}{c_{2}\left(b_{d} \lambda^{n}+c_{2}\right)}\right] .
$$

In order to prove an upper bound, write

$$
\begin{array}{r}
\mathbf{P}^{0}\left\{\exists n: C_{0}^{n}(0)=\emptyset\right\} \leq \mathbf{P}^{0}\left\{r_{\infty}(0)=0\right\} \\
\leq \mathbf{P}^{0}\left\{r_{\infty}(0)=0 \mid r_{0}(0)>r\right\}+\mathbf{P}^{0}\left\{r_{0}(0) \leq r\right\},
\end{array}
$$


for arbitrary $r>0$. The event $\left\{r_{0}(0)>r\right\}$ means that the ball $b(0,2 r)$ contains no points of $\Pi_{0}$, therefore its probability equals $\exp \left\{-b_{d} 2^{d} r^{d}\right\}$. Using the estimate (7) of Theorem 2 , for $r>c \sqrt{d}$, we finally get

$$
\mathbf{P}^{0}\left\{C_{0}^{N}(0)=\emptyset\right\} \leq 1-\sup _{r \geq c \sqrt{d}}\left[\exp \left\{-b_{d} 2^{d} r^{d}\right\}\left(1-a_{1} \sum_{n=1}^{\infty}\left(\frac{\phi\left(\lambda_{n}\right)}{\lambda_{n}}\right)^{A(\rho)}\right)\right],
$$

and in the exponential case, with $r_{*}=c_{*}$,

$$
\mathbf{P}^{0}\left\{C_{0}^{N}(0)=\emptyset\right\} \leq 1-\sup _{r \geq c_{*}}\left[\exp \left\{-b_{d} 2^{d} r^{d}\right\}\left(1-c_{1} \exp \left\{-c_{2} r^{d}\right\}\right)\right] .
$$

\section{Limit tessellation}

Heuristic arguments suggest that the difference between two successive aggregate cells becomes smaller and smaller if the intensities of the point processes grow sufficiently fast. One might expect, and Corollary 4 proves this, that with positive probability the family of cells $\left\{C_{0}^{n}\left(x_{i}^{0}\right)\right\}$ centered in the same nucleus $x_{i}^{0}$ possesses a non-empty "core" int $\left(\cap_{n} C_{0}^{n}(0)\right)$. However, a priori it is unclear if the boundaries of the cells stabilize as $n \rightarrow \infty$. Therefore, it is important to know, whether there exists and in what sense a limit object for the process of tessellations, and if the answer is positive, whether this limit object is itself a tessellation. In this section we will address these questions.

Define the limit cell $C_{0}^{\infty}\left(x_{i}^{0}\right)$ as

$$
C_{0}^{\infty}\left(x_{i}^{0}\right)=\operatorname{cl}\left(\cup_{m} \cap_{n \geq m} C_{0}^{n}\left(x_{i}^{0}\right)\right) .
$$

Thus $C_{0}^{\infty}\left(x_{i}^{0}\right)$ is the closure of those $x$ that belong to $C_{0}^{n}\left(x_{i}^{0}\right)$ for all $n$ starting from some $m$. Our main purpose is to show that such defined objects under a suitable condition constitute a tessellation in $\mathbb{R}^{d}$.

Theorem 3 Assume that a sequence of stationary aggregate tessellations satisfies

$$
\mathbf{P}\left\{\exists x_{i}^{0} \in \Pi_{0}: 0 \in \operatorname{int}\left(\cap_{n=0}^{\infty} C_{0}^{n}\left(x_{i}^{0}\right)\right)\right\}>0 .
$$

Then the sets $\left\{C_{0}^{\infty}\left(x_{i}^{0}\right)\right\}$ a.s. constitute a tessellation of $\mathbb{R}^{d}$.

To prove this theorem we need the following lemma.

Lemma 1 Assume that a sequence of stationary aggregate tessellations satisfies condition (22). Then for any $y \in \mathbb{R}^{d}$ there exists an almost surely unique limit cell $C_{0}^{\infty}\left(x_{i}^{0}\right)$ such that $y \in \operatorname{int}\left(C_{0}^{\infty}\left(x_{j}^{0}\right)\right)$.

INRIA 
Proof. Because of the stationarity, it is sufficient to prove the Lemma for $y=0$. Let $C_{m}^{n}\left(x_{i}^{m}\right)$ be the cells of the aggregate tessellation $\Theta_{m}^{n}$ defined as

$$
\Theta_{m}^{n}=\Theta^{m} \circ \Theta^{m+1} \circ \ldots \circ \Theta^{n} .
$$

Denote by $x^{n}(0)$ the point of $\Pi_{n}$ such that $0 \in C^{n}\left(x^{n}(0)\right)$ and consider the following r.v.'s:

$$
\begin{aligned}
& T_{1}=\min \left\{n>0: 0 \notin \operatorname{int} \cap_{k=0}^{n} C_{0}^{k}\left(x^{0}(0)\right)\right\}, \\
& T_{2}=\min \left\{n>T_{1}: 0 \notin \operatorname{int} \cap_{k=T_{1}}^{n} C_{T_{1}}^{k}\left(x^{T_{1}}(0)\right)\right\}, \\
& \ldots \\
& T_{n}=\min \left\{n>T_{n-1}: 0 \notin \operatorname{int} \cap_{k=T_{n-1}}^{n} C_{T_{n-1}}^{k}\left(x^{T_{n-1}}(0)\right)\right\},
\end{aligned}
$$

Thus $T_{n}$ are the levels at which the aggregate cell containing the origin changes. Clearly, if some $T_{i}=\infty$ then $T_{j}=\infty$ for all $j \geq i$. By assumption (22), we have $\mathbf{P}\left\{T_{1}<\infty\right\}=p>0$. Therefore, for all $n>1$,

$$
\mathbf{P}\left\{T_{n}<\infty\right\} \leq \mathbf{P}\left\{T_{1}<\infty\right\}=p .
$$

Moreover, $T_{n}$ are stopping times and the distribution of $T_{n+1}$ given $\left\{T_{n}=k\right\}$ depends only on $\Pi_{k+1}, \Pi_{k+2}, \ldots$. Therefore,

$$
\mathbf{P}\left\{T_{1}<\infty, \ldots, T_{n}<\infty\right\} \leq p^{n} .
$$

Thus $\mathbf{P}\left\{\forall k, T_{k}<\infty\right\}=0$ so that a.s. there exists $n \geq 0$ such that $T_{n}<\infty$, but $T_{n+1}=\infty$. The lemma statement now holds for a.s. unique $x_{i}^{0}$ such that $x^{n}(0) \in C_{0}^{n}\left(x_{i}^{0}\right)$.

Proof of Theorem 3. Recall the definition of a tessellation from Section 1. We start from verifying condition (b). By Lemma 1 , with probability 1 , for every $i \neq j$,

$$
\operatorname{int}\left(C_{0}^{\infty}\left(x_{i}^{0}\right)\right) \cap \operatorname{int}\left(C_{0}^{\infty}\left(x_{j}^{0}\right)\right)=\emptyset .
$$

Otherwise, with positive probability for some $i \neq j$,

$$
\left|\operatorname{int}\left(C_{0}^{\infty}\left(x_{i}^{0}\right)\right) \cap \operatorname{int}\left(C_{0}^{\infty}\left(x_{j}^{0}\right)\right)\right|>0,
$$

and hence with positive probability a point $y \in \mathbb{R}^{d}$ is covered by the intersection of the interiors of the two cells. This contradicts the uniqueness in Lemma 1. Consequently, for the boundary $\Gamma_{0}^{\infty}$ of the limit tessellation one has

$$
\left|\Gamma_{0}^{\infty}\right|=\left|\mathbb{R}^{d} \backslash \cup_{i} \operatorname{int}\left(C_{0}^{\infty}\left(x_{i}^{0}\right)\right)\right|=0 \quad \text { a.s . }
$$


Let us verify (c). For a Borel set $B \subset \mathbb{R}^{d}$ introduce a random variable

$$
N(B)=\limsup _{n \rightarrow \infty} \sum_{x_{i}^{0} \in \Pi_{0}} \mathbb{1}\left\{C_{0}^{n}\left(x_{i}^{0}\right) \cap B \neq \emptyset\right\}
$$

Obviously, $N(B)=\infty$ if an infinite number of cells $C_{0}^{\infty}\left(x_{i}^{0}\right)$ intersects $B$. If $\sigma_{n}$ denotes the sigma-algebra generated by the sequence of processes $\Pi_{k}, k=n, \ldots, \infty$ then the event $\{N(B)=\infty\}$ belongs to the tail sigma-algebra $\sigma_{\infty}=\cap_{n} \sigma_{n}$. Since $\Pi_{k}$ are independent, the zero-one law applies so that $\mathbf{P}\{N(B)=\infty\}=0$ or 1 . From (22) it follows that for some $\varepsilon>0$

$$
\mathbf{P}\left\{\exists x_{i}^{0} \in \Pi_{0}: b(0, \varepsilon) \in \operatorname{int}\left(\cap_{n=0}^{\infty} C_{0}^{n}\left(x_{i}^{0}\right)\right)\right\}>0,
$$

and hence, $\mathbf{P}\{N(b(0, \varepsilon))=\infty\}=0$. Every bounded set $B \subset \mathbb{R}^{d}$ can be covered by a finite family $\left\{b\left(t_{k}, \varepsilon\right)\right\}_{k<K}$ of copies of $b(0, \varepsilon)$ shifted by $t_{k}$. Because of stationarity, $N(b(0, \varepsilon))$ and $N\left(b\left(t_{k}, \varepsilon\right)\right)$ have the same distribution for each $t_{k} \in \mathbb{R}^{d}$, therefore

$$
\mathbf{P}\{N(B)=\infty\} \leq \sum_{k=1}^{K} \mathbf{P}\left\{N\left(b\left(t_{k}, \varepsilon\right)\right)=\infty\right\}=0 .
$$

In order to prove (a), we need to show that the set

$$
\Phi=\mathbb{R}^{d} \backslash \cup_{i} C_{0}^{\infty}\left(x_{i}^{0}\right)
$$

is a.s. empty. Observe that $\Phi \subset \Gamma_{0}^{\infty}$, and therefore $\Phi$ contains only boundary points if non-empty. For such a point $y \in \Phi$, there exists a sequence

$$
\left\{y_{k}\right\} \subset \cup_{i} C_{0}^{\infty}\left(x_{i}^{0}\right)
$$

converging to $y$. Being itself a bounded set, this sequence visits only a finite number of limit cells $C_{0}^{\infty}\left(x_{i}^{0}\right)$. At least one of these cells contains an infinite subsequence $\left\{y_{k_{n}}\right\}$, and hence it contains $y$ because cells are closed sets. We come to a contradiction with the non-emptiness of $\Phi$.

Corollary 6 Assume that the intensities $\left\{\lambda_{n}\right\}$ for PVAT satisfy condition (5). Then the family $\left\{C_{0}^{\infty}\left(x_{i}^{0}\right)\right\}$ constitutes a tessellation of $\mathbb{R}^{d}$.

Proof. In view of Corollary 4, the condition (22) is satisfied and thus Theorem 3 applies.

Remark 2 Examining the proof of Lemma 1 one may see that a stronger result is proved there: for all $y \in \mathbb{R}^{d}$

$$
\mathbf{P}\left\{\exists x_{i}^{0} \in \Pi_{0}: y \in \cup_{m} \text { int } \cap_{m \geq n} C_{0}^{n}\left(x_{i}^{0}\right)\right\}=1 .
$$


One always has

$$
\cup_{m} \operatorname{int} \cap_{m \geq n} C_{0}^{n}\left(x_{i}^{0}\right) \subseteq \operatorname{int} \cup_{m} \cap_{m \geq n} C_{0}^{n}\left(x_{i}^{0}\right) \subseteq \operatorname{int} C_{0}^{\infty}\left(x_{i}^{0}\right) .
$$

Replacing int $\left(C_{0}^{\infty}\left(x_{i}^{0}\right)\right)$ with $\cup_{m}$ int $\cap_{m \geq n} C_{0}^{n}\left(x_{i}^{0}\right)$ in the proof of part (b) of Theorem 3 yields

$$
\mid \mathbb{R}^{d} \backslash \cup_{i}\left(\cup_{m} \text { int } \cap_{m \geq n} C_{0}^{n}\left(x_{i}^{0}\right)\right) \mid=0
$$

instead of (23). So, in fact, one has almost surely

$$
\cup_{m} \operatorname{int} \cap_{m \geq n} C_{0}^{n}\left(x_{i}^{0}\right)=\operatorname{int} \cup_{m} \cap_{m \geq n} C_{0}^{n}\left(x_{i}^{0}\right)=\operatorname{int} C_{0}^{\infty}\left(x_{i}^{0}\right) .
$$

It is possible to define the limit tessellations in various other ways. Let us show that the most natural ones are essentially equivalent in that they define the same tessellation.

Let $\left\{A_{n}\right\}$ be a sequence of closed subsets of $\mathbb{R}^{d}$. Recall the definitions of the lower and upper set limits:

$$
\begin{aligned}
\liminf A_{n} & =\left\{x: \exists\left\{x_{n}\right\} \text { such that } x_{n} \in A_{n} \text { and } x=\lim _{n} x_{n}\right\}, \\
\lim \sup A_{n} & =\left\{x: \exists\left\{x_{n_{k}}\right\} \text { such that } x_{n_{k}} \in A_{n_{k}} \text { and } x=\lim _{k} x_{n_{k}}\right\} .
\end{aligned}
$$

In words, a point belongs to $\lim \inf A_{n}$ if and only if any its neighborhood intersects with all sets $A_{n}$ starting from some $m$; a point belongs to $\lim \sup A_{n}$ if and only if any its neighborhood intersects infinitely many sets $A_{n}$. Both limits are closed sets (see, e. g., [8, Prop. 1.2.3]). Define the sets

$$
\begin{aligned}
D_{0}^{\infty}\left(x_{i}^{0}\right) & =\operatorname{cl}\left(\cap_{m} \cup_{n \geq m} C_{0}^{n}\left(x_{i}^{0}\right)\right), \\
\underline{E}\left(x_{i}^{0}\right) & =\underset{n}{\lim \inf } C_{0}^{n}\left(x_{i}^{0}\right), \\
\bar{E}\left(x_{i}^{0}\right) & =\underset{n}{\lim \sup _{0}} C_{0}^{n}\left(x_{i}^{0}\right) .
\end{aligned}
$$

Corollary 7 Assume that a sequence of stationary aggregate tessellations satisfies condition (22). Then the sets $C_{0}^{\infty}\left(x_{i}\right), D_{0}^{\infty}\left(x_{i}^{0}\right), \underline{E}\left(x_{i}^{0}\right)$, and $\bar{E}\left(x_{i}^{0}\right)$ defined by (21), (25-27) have almost surely the same interior, may differ only on the set of a null measure and thus define the same tessellation.

Proof. Obviously, $C_{0}^{\infty}\left(x_{i}^{0}\right) \subseteq D_{0}^{\infty}\left(x_{i}^{0}\right) \subseteq \bar{E}\left(x_{i}^{0}\right)$ and $C_{0}^{\infty}\left(x_{i}^{0}\right) \subseteq \underline{E}\left(x_{i}^{0}\right) \subseteq \bar{E}\left(x_{i}^{0}\right)$ Moreover, $\bar{E}\left(x_{i}^{0}\right) \cap \operatorname{int}\left(C_{0}^{\infty}\left(x_{j}^{0}\right)\right)=\emptyset$ for any $j \neq i$. Therefore,

$$
\begin{aligned}
& D_{0}^{\infty}\left(x_{i}^{0}\right) \backslash C_{0}^{\infty}\left(x_{i}^{0}\right) \subseteq \bar{E}\left(x_{i}^{0}\right) \backslash C_{0}^{\infty}\left(x_{i}^{0}\right) \subseteq \bar{E}\left(x_{i}^{0}\right) \backslash \operatorname{int} C_{0}^{\infty}\left(x_{i}^{0}\right) \\
& \quad=\bar{E}\left(x_{i}^{0}\right) \backslash \cup_{i} \operatorname{int} C_{0}^{\infty}\left(x_{i}^{0}\right) \subseteq \mathbb{R}^{d} \backslash \cup_{i} \operatorname{int} C_{0}^{\infty}\left(x_{i}^{0}\right)=\Gamma_{0}^{\infty} .
\end{aligned}
$$


Replacing $D_{0}^{\infty}\left(x_{i}^{0}\right)$ by $\underline{E}\left(x_{i}^{0}\right)$, we obtain that the same relation is also true for $\underline{E}\left(x_{i}^{0}\right)$. the assertion now follows from (23).

The following complementary result asserts the convergence of the boundaries

$$
\Gamma_{0}^{n}=\mathbb{R}^{d} \backslash \cup_{i} \operatorname{int}\left(C_{0}^{n}\left(x_{i}^{0}\right)\right) .
$$

of the aggregate tessellations $\Theta_{0}^{n}$ to the the boundary $\Gamma_{0}^{\infty}$ of the limit one. This limit boundary can be a fractal as it is shown in Section 6 for the case of PVAT .

Corollary 8 Assume that a sequence of stationary aggregate tessellations satisfies condition (22). Then

$$
\lim \inf \Gamma_{0}^{n}=\lim \sup \Gamma_{0}^{n}=\Gamma_{0}^{\infty} \quad \text { a.s. }
$$

Also, the distances

$$
d\left(x, \Gamma_{0}^{n}\right)=\inf \left\{\|x-y\|, y \in \Gamma_{0}^{n}\right\}
$$

converge a.s. to $d\left(x, \Gamma_{0}^{\infty}\right)$ for every $x \in \mathbb{R}^{d}$.

Proof. According to [8, Prop. 1.2.5]), the two definitions (28) and (29) of convergence are equivalent in $\mathbb{R}^{d}$. We will first show that $\Gamma_{0}^{\infty} \subset \liminf \Gamma_{0}^{n}$. If $x \in \Gamma_{0}^{\infty}$, then by (23), $x \in C_{0}^{\infty}\left(x_{i}^{0}\right) \cap C_{0}^{\infty}\left(x_{j}^{0}\right)$ for some $i \neq j$. Therefore, a neighborhood of $x$ hits $\cap_{n \geq m} C_{0}^{n}\left(x_{i}^{0}\right)$ and $\cap_{n \geq m} C_{0}^{n}\left(x_{j}^{0}\right)$ starting from some $m$. Then it must also hit $\Gamma_{0}^{n}$ for all $n \geq m$.

Let us verify that $\lim \sup \Gamma_{0}^{n} \subset \Gamma_{0}^{\infty}$. Suppose that $x_{n_{k}} \in \Gamma_{0}^{n_{k}}$ and $x=\lim _{k} x_{n_{k}}$. If $x \notin \Gamma_{0}^{\infty}$, then $x \in \operatorname{int}\left(C_{0}^{\infty}\left(x_{i}^{0}\right)\right)$ for some $i$. By $(24), x \in \operatorname{int} \cap_{n \geq m} C_{0}^{n}\left(x_{i}^{0}\right)$ for some $m$, and the sequence of $x_{n_{k}} \in \Gamma_{0}^{n_{k}}$ cannot converge to $x$. From this contradiction it follows that $x \in \Gamma_{0}^{\infty}$.

Corollary 9 Under condition (22), for each $y \in \mathbb{R}^{d}$,

$$
\lim _{n} \mathbf{P}^{0}\left\{y \in C_{0}^{n}(0)\right\}=\mathbf{P}^{0}\left\{y \in C_{0}^{\infty}(0)\right\} .
$$

Proof. By Corollary 7,

$$
\mathbf{P}^{0}\left\{y \in C_{0}^{\infty}(0)\right\}=\mathbf{P}^{0}\left\{y \in D_{0}^{\infty}(0)\right\} .
$$

Using the continuity property of the probability measures, we obtain

$$
\begin{aligned}
& \mathbf{P}^{0}\left\{y \in C_{0}^{\infty}(0)\right\}=\lim _{m} \mathbf{P}^{0}\left\{y \in \cup_{n \geq m} C_{0}^{n}(0)\right\} \geq \lim _{n} \mathbf{P}^{0}\left\{y \in C_{0}^{n}(0)\right\}, \\
& \mathbf{P}^{0}\left\{y \in C_{0}^{\infty}(0)\right\}=\lim _{m} \mathbf{P}^{0}\left\{y \in \cap_{n \geq m} C_{0}^{n}(0)\right\} \leq \lim _{n} \mathbf{P}^{0}\left\{y \in C_{0}^{n}(0)\right\} .
\end{aligned}
$$

INRIA 
Remark 3 Replacing in the last proof $\mathbf{P}^{0}\{y \in \cdot\}$ by $|\cdot|$, we get

$$
\lim _{n}\left|C_{0}^{n}(0)\right|=\left|C_{0}^{\infty}(0)\right| \text { a.s. }
$$

Also,

$$
\mathbf{E}^{0}\left|C_{0}^{\infty}(0)\right|=1
$$

due to the stationarity of the tessellation.

\section{Spherical contact distribution function of the boundary}

From now on we confine ourselves to PVAT with exponentially growing intensities. As we have shown in the previous section, if (5) holds, the limit Poisson-Voronoi tessellations exist and the boundary of the limit cells $\Gamma_{0}^{\infty}$ is a random closed set defined by (23). One of its important characteristics is the spherical contact distribution function $H(r)$, defined as

$$
H(r)=\mathbf{P}\left\{\Gamma_{0}^{\infty} \cap b(0, r) \neq \emptyset \mid 0 \notin \Gamma_{0}^{\infty}\right\}, \quad r \geq 0 .
$$

Here, as Lemma 1 shows, the probability of the condition is one, thus $H(0)=0$. Some information on the degree of variability of the cell boundary can be derived from the rate at which $H(r)$ decreases as $r$ tends to zero. The aim of this section is to prove the following result.

Theorem 4 For PVAT with exponentially growing intensities: $\lambda_{n}=\lambda^{n}$ for some $\lambda>1$, there exist constants $K>0$ and $q \in(0,1)$ such that for all $r \geq 0$,

$$
H(r)=\mathbf{P}\left\{b(0, r) \cap \Gamma_{0}^{\infty} \neq \emptyset\right\} \leq K r^{d q} .
$$

The values of $q$ and $K$ are given in (37).

Proof. Consider the cells of the limit tessellation $\left\{C_{n}^{\infty}\left(x_{i}^{n}\right)\right\}$ defined in the same way as in (21). Let $X(n, r)$ be the nuclei of those cells whose boundary crosses the ball $b(0, r)$, that is

$$
X(n, r)=\left\{x_{i}^{n} \in \Pi_{n}: \partial C_{n}^{\infty}\left(x_{i}^{n}\right) \cap b(0, r) \neq \emptyset\right\} .
$$

We will first prove the estimate: for each $n \geq 1$ and for each $s>0$,

$$
\mathbf{P}\left\{b(0, r) \cap \Gamma_{0}^{\infty} \neq \emptyset\right\}<f(r, n, s)^{n},
$$

where

$$
f(r, n, s)=1-\mathbf{P}\{X(n, r) \subset b(0, s)\} \mathbf{P}\left\{b(0, s) \subset C^{n}\left(x^{n-1}(0)\right)\right\} .
$$


Recall that $x^{n-1}(0)$ denotes the closest to 0 point of $\Pi_{n-1}$. Consider the events

$$
E(m, r)=\left\{b(0, r) \cap \Gamma_{m}^{\infty} \neq \emptyset\right\},
$$

where $\Gamma_{m}^{\infty}$ is the boundary of the tessellation $\left\{C_{m}^{\infty}\left(x_{i}^{m}\right)\right\}$. Note that $E(0, r)$ is the event in the left-hand side of (30). Since $\Gamma_{m}^{\infty} \subset \Gamma_{m+1}^{\infty}$, we have $E(m, r) \subset E(m+1, r)$ and therefore,

$$
\begin{aligned}
\mathbf{P}\{E(0, r)\}=\mathbf{P}\{E(1, r)\} & \mathbf{P}\{E(0, r) \mid E(1, r)\} \\
=\mathbf{P}\{E(n, r)\} \prod_{m=1}^{n} \mathbf{P}\{E(m-1, r) \mid E(m, r)\} & <\prod_{m=1}^{n} \mathbf{P}\{E(m-1, r) \mid E(m, r)\} .
\end{aligned}
$$

For every $m$ and $s_{m}>0$,

$$
\mathbf{P}\{E(m-1, r) \mid E(m, r)\} \leq f\left(r, m, s_{m}\right),
$$

Indeed, if $b(0, r) \cap \Gamma_{m}^{\infty} \neq \emptyset$ and

$$
X(m, r) \subset b\left(0, s_{m}\right) \subset C^{m-1}\left(x^{m-1}(0)\right)
$$

then the cells $C_{m}^{\infty}\left(x_{i}^{m}\right)$ for which $x_{i}^{m} \in X(m, r)$ join in $C_{m-1}^{\infty}\left(x^{m-1}(0)\right)$ so that $b(0, r) \cap$ $\Gamma_{m-1}^{\infty}=\emptyset$.

Since the intensity of each $\Pi_{n}$ equals $\lambda^{n}$, the distributions of

$$
\left(\Pi_{n}, \Pi_{n+1}, \ldots\right) \quad \text { and } \quad\left(\lambda^{1 / d} \Pi_{n+1}, \lambda^{1 / d} \Pi_{n+2}, \ldots\right)
$$

coincide. Consequently, the sets $X(m+1, r)$ and $\lambda^{-1 / d} X\left(m, \lambda^{1 / d} r\right)$ have the same distribution, and

$$
\begin{aligned}
\mathbf{P}\left\{X(m+1, r) \subset b\left(0, s_{m}\right)\right\} & =\mathbf{P}\left\{\lambda^{-1 / d} X\left(m, \lambda^{1 / d} r\right) \subset b\left(0, s_{m}\right)\right\} \\
& =\mathbf{P}\left\{X\left(m, \lambda^{1 / d} r\right) \subset b\left(0, \lambda^{1 / d} s_{m}\right)\right\} \\
& <\mathbf{P}\left\{X(m, r) \subset b\left(0, \lambda^{1 / d} s_{m}\right)\right\}
\end{aligned}
$$

as $X(m, r) \subseteq X\left(m, \lambda^{1 / d} r\right)$. Also

$$
\mathbf{P}\left\{b\left(0, s_{m}\right) \subset C^{m}\left(x^{m}(0)\right\}=\mathbf{P}\left\{b\left(0, \lambda^{1 / d} s_{m}\right) \subset C^{m-1}\left(x^{m-1}(0)\right)\right\}\right.
$$

so that $f\left(r, m-1, \lambda^{1 / d} s_{m}\right)<f\left(r, m, s_{m}\right)$. Alternatively, $f\left(r, m, s_{m}\right)<f\left(r, m+1, \lambda^{-1 / d} s_{m}\right)<$ $f\left(r, n, \lambda^{-(n-m) / d} s_{m}\right)$ by induction. Thus, taking $s_{m}=\lambda^{(n-m) / d} s$ in (32), by (31) we obtain (30).

INRIA 
Next, we find a bound for $f(r, n, s)$. We have

$$
\begin{gathered}
1-\mathbf{P}\{X(n, r) \subset b(0, s)\}=1-\mathbf{P}\left\{X(0, r) \subset b\left(0, \lambda^{n / d} s\right)\right\} \\
<\mathbf{P}\left\{\exists x_{i}^{0}: x_{i}^{0} \notin b\left(0, \lambda^{n / d} s\right) \text { and } C_{0}^{\infty}\left(x_{i}^{0}\right) \cap b\left(0, \lambda^{n / d} r\right) \neq \emptyset\right\} \\
<\mathbf{P}\left\{\exists x_{i}^{0}: x_{i}^{0} \notin b\left(0, \lambda^{n / d} s\right) \text { and } R_{\infty}\left(x_{i}^{0}\right)>\left\|x_{i}^{0}\right\|-\lambda^{n / d} r\right\} \\
<\mathbf{E} \sum_{x_{i}^{0} \in \Pi_{0}} \mathbb{I}\left(\left\|x_{i}^{0}\right\|>\lambda^{n / d} s ; R_{\infty}\left(x_{i}^{0}\right)>\left\|x_{i}^{0}\right\|-\lambda^{n / d} r\right) .
\end{gathered}
$$

By the Campbell theorem, the last expectation equals

$$
\begin{aligned}
& \int_{\|z\|>\lambda^{n / d} s} \mathbf{P}^{0}\left\{R_{\infty}(0)>\|z\|-\lambda^{n / d} r\right\} d z \\
&=\lambda^{n} \int_{\|z\|>s} \mathbf{P}^{0}\left\{R_{\infty}(0)>\lambda^{n / d}(\|z\|-r)\right\} d z
\end{aligned}
$$

Assume that $s$ and $n$ satisfy the inequality

$$
\lambda^{n / d}(s-r) \geq c_{*},
$$

with $c_{*}$ as in Corollary 1 . Then by (13), the right-hand side of (33) is smaller than

$$
c_{1} \lambda^{n} \int_{\|z\|>s} e^{-c_{2} \lambda^{n}(\|z\|-r)^{d}} d z=c_{1} \lambda^{n} \int_{s}^{\infty} d b_{d} \rho^{d-1} e^{-c_{2} \lambda^{n}(\rho-r)^{d}} d \rho \leq\left(c_{1} / c_{2}\right) e^{-c_{2} \lambda^{n}(s-r)^{d}}
$$

and therefore,

$$
\mathbf{P}\{X(n, r) \subset b(0, s)\}>1-\left(c_{1} / c_{2}\right) e^{-c_{2} \lambda^{n}(s-r)^{d}} .
$$

Also, since there is only one Voronoi cell that may contain a ball, we may write

$$
\begin{aligned}
\mathbf{P}\left\{b(0, s) \subset C^{n-1}\left(x^{n-1}(0)\right)\right\} & =\mathbf{P}\left\{b\left(0, \lambda^{(n-1) / d} s\right) \subset C^{0}\left(x^{0}(0)\right)\right\} \\
& =\mathbf{E} \sum_{x_{i}^{0} \in \Pi_{0}} \mathbb{I}\left\{b\left(0, \lambda^{(n-1) / d} s\right) \subset C^{0}\left(x_{i}^{0}\right)\right\} \\
& =\int \mathbf{P}^{0}\left\{b\left(z, \lambda^{(n-1) / d} s\right) \subset C^{0}(0)\right\} d z .
\end{aligned}
$$

Using (16), we obtain

$$
\mathbf{P}\left\{b(0, s) \subset C^{n-1}\left(x^{n-1}(0)\right)\right\}>b_{d} \lambda^{n-1} s^{d} e^{-b_{d} \lambda^{n-1}(3 s)^{d}} .
$$


Combining (35) and (36), for such $s$ and $n$ that (34) holds, we get

$$
\begin{aligned}
\mathbf{P}\left\{b(0, r) \cap \Gamma_{0}^{\infty} \neq \emptyset\right\}<\left[1-\left(1-\frac{c_{1}}{c_{2}} \exp \left\{-c_{2} \lambda^{n}(s-r)^{d}\right\}\right)\right. & \\
& \left.\times b_{d} \lambda^{n-1} s^{d} \exp \left\{-b_{d} \lambda^{n-1}(3 s)^{d}\right\}\right]^{n} .
\end{aligned}
$$

One of the possible choices of the constants $s$ and $n$ is

$$
s=2 r \quad \text { and } \quad n=d \log _{\lambda}\left(c_{*} / r\right) .
$$

Then

$$
\mathbf{P}\left\{b(0, r) \cap \Gamma_{0}^{\infty} \neq \emptyset\right\}<K r^{d q},
$$

where

$$
\begin{aligned}
q & =-\log _{\lambda}\left[1-\left(1-\frac{c_{1}}{c_{2}} e^{-c_{2} c_{*}^{d}}\right) \frac{b_{d}\left(2 c_{*}\right)^{d}}{\lambda} e^{-b_{d}\left(6 c_{*}\right)^{d} / \lambda}\right] \\
K & =c_{*}^{-d q} .
\end{aligned}
$$

Since $\lambda>1$, we have $0<q<1$. The theorem is proved.

\section{$6 \quad$ Fractal boundary of the limit cells}

In the case of exponentially growing intensities, the distributions of $\Gamma_{0}^{\infty}$ and $\Gamma_{n}^{\infty}$ scaled by $\lambda^{n / d}$ coincide, i.e., the boundary of the limit tessellation is statistically self-similar. This property is rather different from geometrical self-similarity in the sense of $\Gamma_{0}^{\infty}$ being a union of scaled copies of self. However, by construction, $\Gamma_{n-1}^{\infty}$ consists of parts of $\Gamma_{n}^{\infty}$, and therefore, $\Gamma_{0}^{\infty}$ has a similar structure at any scale of observation, which allows us to call it a fractal.

The primary characteristic of a fractal is its dimension, which can be defined in several ways. We will be interested in the Hausdorff dimension of $\Gamma_{0}^{\infty}$ (see, e. g., [5, p. 20-23] for definitions of different dimensions that we use here).

Theorem 5 Let $q$ be the constant defined in (37). Then for PVAT with exponentially growing intensities: $\lambda_{n}=\lambda^{n}$ for some $\lambda>1$, one has

$$
\begin{aligned}
& \operatorname{dim}_{H} \Gamma_{0}^{\infty}=\mathbf{E} \operatorname{dim}_{H} \Gamma_{0}^{\infty} \quad \text { a.s.; } \\
& \operatorname{dim}_{H} \Gamma_{0}^{\infty}<d(1-q) \quad \text { a.s. }
\end{aligned}
$$

INRIA 
Proof. Consider the collection of mesh cubes of size $M$ in $\mathbb{R}^{d}$ and let $\left\{\theta_{\alpha}\right\}$ be the family of shifts translating the cube at the origin $[0, M)^{d}$ by the vector $M \alpha$, where $\alpha=\left(\alpha_{1}, \ldots, \alpha_{d}\right) \in$ $\mathbb{Z}^{d}$. Introduce also

$$
\begin{aligned}
\Lambda_{N} & =\left\{\alpha:\left|\alpha_{i}\right| \leq N, i=1, \ldots, d\right\} \\
\Gamma_{0}^{\infty}(M) & =\Gamma_{0}^{\infty} \cap[0, M)^{d} \\
\theta_{\alpha} \Gamma_{0}^{\infty}(M) & =\Gamma_{0}^{\infty} \cap \theta_{\alpha}[0, M)^{d} .
\end{aligned}
$$

Since $\theta_{\alpha} \Gamma_{0}^{\infty}(M) \subset \Gamma_{0}^{\infty}$, with probability 1 for all $\alpha$, we have

$$
\operatorname{dim}_{H} \Gamma_{0}^{\infty} \geq \operatorname{dim}_{H} \theta_{\alpha} \Gamma_{0}^{\infty}(M),
$$

and hence

$$
\operatorname{dim}_{H} \Gamma_{0}^{\infty} \geq \sup _{\alpha \in \Lambda_{N}} \operatorname{dim}_{H} \theta_{\alpha} \Gamma_{0}^{\infty}(M)
$$

Now by the ergodic theorem,

$$
\begin{aligned}
\operatorname{dim}_{H} \Gamma_{0}^{\infty} & \geq \lim _{N \rightarrow \infty} \sup _{\alpha \in \Lambda_{N}} \operatorname{dim}_{H} \theta_{\alpha} \Gamma_{0}^{\infty}(M) \\
& \geq \lim _{N \rightarrow \infty} \frac{1}{(2 N)^{d}} \sum_{\alpha \in \Lambda_{N}} \operatorname{dim}_{H} \theta_{\alpha} \Gamma_{0}^{\infty}(M) \\
& =\mathbf{E} \operatorname{dim}_{H} \Gamma_{0}^{\infty}(M) .
\end{aligned}
$$

Letting $M \rightarrow \infty$ in this inequality and using the property of monotonicity of the Hausdorff dimension, we get

$$
\operatorname{dim}_{H} \Gamma_{0}^{\infty} \geq \mathbf{E} \operatorname{dim}_{H} \Gamma_{0}^{\infty},
$$

which implies the first equality of the theorem.

To prove the second inequality, we make use of the estimate of the Hausdorff dimension of a set by its upper box dimension (see, e. g., [5, p. 24]). Let $N_{\varepsilon}(B)$ be the smallest number of closed balls of radius $\varepsilon$ that cover $B$. Then

$$
\operatorname{dim}_{H} \Gamma_{0}^{\infty}(M) \leq \limsup _{\varepsilon \rightarrow 0} \frac{\log N_{\varepsilon}\left(\Gamma_{0}^{\infty}(M)\right)}{-\log \varepsilon} .
$$

Take expectations at both sides of this inequality. It can be easily verified that $[0, M)^{d}$, and hence $\Gamma_{0}^{\infty}(M)$, can be covered by a family $\left\{b_{i}\right\}$ of less than $(M \sqrt{d} / 2 \varepsilon)^{d}$ balls of radius $\varepsilon$. Thus the function in the right-hand side under the limit is bounded by a constant not depending on $\varepsilon$, and therefore we can exchange the limit and the expectation. Moreover, the function $\log (\cdot)$ is concave, hence

$$
\mathbf{E} \log (\cdot) \leq \log \mathbf{E}(\cdot)
$$


Therefore,

$$
\mathbf{E} \operatorname{dim}_{H} \Gamma_{0}^{\infty}(M) \leq \limsup _{\varepsilon \rightarrow 0} \frac{\log \mathbf{E} N_{\varepsilon}\left(\Gamma_{0}^{\infty}(M)\right)}{-\log \varepsilon} .
$$

Recalling the definition of the contact distribution $H(r)$ from the previous section, we get

$$
\left.\mathbf{E} N_{\varepsilon}\left(\Gamma_{0}^{\infty}(M)\right) \leq \mathbf{E} \sum_{i} \mathbb{I}\left(b_{i} \cap \Gamma_{0}^{\infty}(M)\right) \neq \emptyset\right) \leq\left(\frac{M \sqrt{d}}{2 \varepsilon}\right)^{d} H(\varepsilon) .
$$

From Theorem 4 it follows that

$$
\mathbf{E} \operatorname{dim}_{H} \Gamma_{0}^{\infty}(M) \leq \limsup _{\varepsilon \rightarrow 0} \frac{d \log (M \sqrt{d} / 2)-d \log \varepsilon+\log K+d q \log \varepsilon}{-\log \varepsilon}=d(1-q),
$$

and it remains to let $M \rightarrow \infty$ to obtain the second statement of the theorem.

\section{References}

[1] F. Baccelli, M. Klein, M. Lebourges, and S. Zuyev. Stochastic geometry and architecture of communication networks. J. Telecommunication Systems, 7:209-227, 1997.

[2] F. Baccelli and S. Zuyev. Stochastic geometry models of mobile communication networks. In Frontiers in queueing, pages 227-243. CRC, Boca Raton, FL, 1997.

[3] F. Baccelli and S. Zuyev. Poisson-Voronoi spanning trees with applications to the optimization of communication networks. Oper. Res., 47(4), 1999.

[4] A. A. Borovkov. Teoriya veroyatnostei [In Russian]. Probability Theory. "Nauka", Moscow, 1986.

[5] K. Falconer. Techniques in fractal geometry. John Wiley \& Sons, Chichester, 1997.

[6] S. G. Foss and S. A. Zuyev. On a Voronoi aggregative process related to a bivariate Poisson process. Adv. in Appl. Probab., 28(4):965-981, 1996.

[7] N. N. Lebedev. Special functions and their applications. Dover Publications Inc., New York, 1972.

[8] G. Matheron. Random sets and integral geometry. John Wiley \& Sons, New YorkLondon-Sydney, 1975.

[9] J. Møller. Random tessellations in $R^{d}$. Adv. in Appl. Probab., 21(1):37-73, 1989.

[10] A. Okabe, B. Boots, and K. Sugihara. Spatial tessellations: concepts and applications of Voronoi diagrams. John Wiley \& Sons Ltd., Chichester, 1992.

[11] D. Stoyan, W. Kendall, and J. Mecke. Stochastic Geometry and its Applications. Wiley, Chichester, 1995. 